ORIGINAL

\title{
Factores asociados a ingreso en unidad de cuidados intensivos en pacientes hospitalizados por Influenza pandémica A/H1N1 2009
}

\author{
A.E. González-Vélez*, C. Díaz-Agero-Pérez, A. Robustillo-Rodela, \\ A.M. Cornejo-Gutiérrez, M.J. Pita-López, L. Oliva-Iñiguez y V. Monge-Jodra
}

Servicio de Medicina Preventiva, Hospital Universitario Ramón y Cajal, Madrid, España

Recibido el 17 de enero de 2011; aceptado el 11 de marzo de 2011

Disponible en Internet el 4 de mayo de 2011

\section{PALABRAS CLAVE \\ Influenza; \\ Factores de riesgo; \\ Complicaciones}

\begin{abstract}
Resumen
Objetivo: Determinar los factores de riesgo para ingreso en la unidad de cuidados intensivos (UCI) en pacientes con infección por virus pandémico (H1N1) 2009.

Diseño: Estudio de cohorte retrospectivo en pacientes ingresados por Influenza A/H1N1 2009 durante el periodo pandémico.

Ámbito: Hospital Universitario Ramón y Cajal.

Pacientes: Todos los pacientes ingresados con reacción en cadena de la polimerasa en transcripción inversa (RT-PCR) positiva para virus de Influenza A/H1N1 2009.

Variables de interés: Historia de factores de riesgo para Influenza grave, vacunación para Influenza estacional 2008-2009, síntomas y signos clínicos, pruebas de laboratorio, hallazgos en la radiografía de tórax, tiempo en la administración de antiviral y estancia hospitalaria.

Resultados: La mediana de edad de 100 casos fue 38 años (mínimo: 4 meses, máximo: 80 años). El 77\% tuvo al menos un factor de riesgo, siendo el asma la comorbilidad más frecuente en los menores de 18 años y el hábito tabáquico en los mayores. La mediana de tiempo entre el comienzo de los síntomas y el inicio de antiviral fue 3 días (mínimo: 0 días, máximo: 18 días). El $19 \%$ de los pacientes fueron ingresados en UCI y el $2 \%$ fallecieron por gripe. En el análisis multivariable, enfermedad metabólica y presencia de infiltrados en la radiografía de tórax se asociaron de forma significativa a ingreso en la UCl.

Conclusión: Una radiografía de tórax anormal en el momento del ingreso, junto con la presencia de ciertas comorbilidades, especialmente enfermedades metabólicas, sugieren la posibilidad de peor pronóstico de gripe pandémica (H1N1) 2009.
\end{abstract}

(c) 2011 Elsevier España, S.L. y SEMICYUC. Todos los derechos reservados.

\footnotetext{
* Autor para correspondencia.

Correo electrónico: aegonzalezv@gmail.com (A.E. González-Vélez).
} 


\section{KEYWORDS}

Influenza;

Human;

Risk factors;

Complications
Factors associated to admission to Intensive Care in patients hospitalized due to pandemic Influenza A/H1N1 2009

\begin{abstract}
Objective: The present study explores the possible factors related to severe cases of pandemic flu.

Design: A retrospective cohort study was conducted in patients hospitalized with Influenza A/H1N1 2009 during the pandemic period.

Setting: Ramon y Cajal University Hospital (Madrid, Spain).

Patients: All hospitalized patients with positive RT-PCR (real-time polymerase chain reaction) for Influenza A/H1N1 2009 virus.

Main variables: The main variables collected were: history of risk factors for severe Influenza, history of immunization, clinical presentation, laboratory tests, chest X-ray report, administration of antiviral treatment, and hospital stay.

Results: The median age of the 100 cases was 38 years (range 4 months to 80 years). Seventyseven percent of the patients had at least one risk factor. Asthma was the most common factor among patients younger than 18 years, versus smoking in the older subjects. Antiviral therapy was initiated a median time of three days (range 0 to 18 days) after the onset of illness. Nineteen percent of the patients were admitted to Intensive Care, and 2\% died. Metabolic disease and abnormal chest X-ray findings were factors associated to admission to the ICU.

Conclusion: As in other studies, abnormal chest $\mathrm{X}$-ray findings upon admission and metabolic disease were related to poor outcomes of 2009 pandemic Influenza $A$ (H1N1) infection in our patients.

๑ 2011 Elsevier España, S.L. and SEMICYUC. All rights reserved.
\end{abstract}

\section{Introducción}

Entre finales de marzo y principios de abril de 2009 las autoridades sanitarias mexicanas detectaron un aumento en el número de casos de Influenza tres veces mayor al registrado en el periodo similar de 2008 1,2 . El 17 de abril, los Centros para el Control y Prevención de Enfermedades (CDC) de Estados Unidos (EE.UU.) confirmaron los primeros 2 casos de infección humana por una nueva cepa de virus Influenza tipificado como A/H1N1 en 2 pacientes pediátricos residentes en California ${ }^{3}$. En España, la circulación del nuevo virus Influenza se detectó por primera vez en la semana del 24 al 30 de mayo de 2009 en la Red de Médicos Centinela de la comunidad de Madrid y 2 semanas después en la Red de Médicos Centinela de Navarra, propagándose posteriormente al resto del territorio nacional ${ }^{4}$. El 11 de junio la Organización Mundial de la Salud elevó su alerta pandémica al nivel más alto, declarando la fase 6 o fase de pandemia, indicando amplia transmisión comunitaria en al menos 2 continentes $^{5}$

La infección por Influenza A/H1N1 2009 produjo una sintomatología leve en la mayoría de los casos, con tasas de ataque más altas en niños y adultos jóvenes, y una letalidad menor al 0,6\%6-12. Sin embargo, las muertes ocurrieron principalmente en población más joven que la afectada por gripe estacional ${ }^{13,14}$. En EE.UU. y Reino Unido, la hospitalización debido a gripe pandémica fue más frecuente en menores de 18 años de edad, particularmente en los menores de un año, y menos frecuente en mayores de 65 años. Más de la mitad de los pacientes hospitalizados presentaba alguna enfermedad de base y entre el 13 y el $44 \%$ había sido ingresado en unidades de cuidados intensivos $(\mathrm{UCI})^{15-17}$.
Varios estudios llevados a cabo en España y en otros países han dado a conocer los factores asociados a riesgo de muerte en los casos de infección por gripe pandémica. La obesidad, el cáncer, las inmunodeficiencias y la enfermedad respiratoria crónica aparecen como los principales factores de riesgo en niños y adultos hospitalizados ${ }^{15,18,19}$. Otros autores también han demostrado asociación entre dichos factores y el riesgo de ingreso en la $\mathrm{UCl}^{16,20,21}$. En España, varias series de pacientes ingresados en $\mathrm{UCI}$ han demostrado que determinadas poblaciones, como los obesos y los pacientes con enfermedad pulmonar crónica, pueden tener un riesgo más elevado de enfermedad grave $^{22,23}$.

Este estudio resume los hallazgos clínicos en pacientes ingresados en el Hospital Universitario Ramón y Cajal para el tratamiento de la infección por Influenza A/H1N1 2009 y explora sus posibles asociaciones con el riesgo de ingreso en la $\mathrm{UCl}$

\section{Pacientes y métodos}

El Hospital Universitario Ramón y Cajal es un centro de tercer nivel con 1.090 camas funcionantes que constituye el hospital de referencia del Área Sanitaria IV de la comunidad de Madrid, atendiendo a una población total de 592.576 habitantes.

Se describen todos los pacientes que fueron ingresados en este hospital, con un cuadro clínico de síndrome gripal (temperatura de $37,8^{\circ} \mathrm{C}$ o mayor y tos o dolor de garganta) o infección respiratoria aguda (aparición súbita menor a $12 \mathrm{~h}$ ) durante al menos $24 \mathrm{~h}$, con reacción en cadena de la polimerasa en transcripción inversa (RT-PCR) positiva para 
virus Influenza A/H1N1 2009 en exudado faríngeo, durante el periodo pandémico.

Los profesionales del servicio de medicina preventiva revisaron las historias clínicas de los pacientes ingresados durante el periodo pandémico, utilizando para ello un formulario estandarizado que incluía información demográfica, historia de factores de riesgo (determinados por el ministerio de Sanidad y Política Social español) y vacunación para Influenza estacional 2008-2009, síntomas y signos clínicos, pruebas de laboratorio, hallazgos en la radiografía de tórax, intervalo entre inicio de síntomas y administración de medicación antiviral, y tiempo de estancia hospitalaria. Se desarrolló un análisis bivariado para comparar las características clínicas de los pacientes y la severidad de la enfermedad, entre aquellos pacientes que no requirieron ingreso en $\mathrm{UCl}$ y aquellos que requirieron ingreso en $\mathrm{UCl}$ o fallecieron por gripe. Se utilizó la prueba no paramétrica $U$ de Mann-Whitney en las variables continuas y las pruebas ji cuadrado o exacta de Fisher en las variables categóricas. Todos los factores que tras este análisis mostraron un valor $\mathrm{p}<0,10$ fueron considerados en un modelo de regresión logística multivariante para determinar las asociaciones entre las características clínicas y la severidad de la enfermedad. La significación estadística fue definida como un valor $\mathrm{p}<0,05$ y todos los análisis fueron llevados a cabo con el software SPSS para Windows versión 15.

\section{Resultados}

\section{Incidencia de hospitalización}

Este estudio describe 100 casos admitidos por síndrome gripal o infección respiratoria aguda.

La tasa global de hospitalización fue de 16,8 casos por cada 100.000 habitantes. Las tasas específicas por grupo de edad revelaron que los menores de 10 años tuvieron la incidencia más alta de hospitalización por la enfermedad, especialmente los menores de 2 años con 69,8 casos por cada 100.000 habitantes, y la más baja entre los 10 y 17 años de edad con 7,5 casos por cada 100.000 habitantes.

\section{Características clínicas}

Los 100 casos incluidos ingresaron en este hospital entre el 7 de julio y el 29 de diciembre de 2009. La mediana de edad de los pacientes fue 38 años (mínimo: 4 meses, máximo: 80 años). La mayoría tenía como lugar de residencia habitual la comunidad de Madrid (94\%) (tabla 1).

Del total de casos incluidos, en $25(25,0 \%)$ pacientes se disponía de información completa acerca de los síntomas iniciales de la enfermedad. Fiebre y malestar general estuvieron presentes en más del $90 \%$ de éstos, seguidos por tos en el $88 \%$, disnea en el $48 \%$ y síntomas como dolor de garganta, rinorrea y cefalea entre el 20 y el $40 \%$ de los casos. La mediana de tiempo entre el inicio de los síntomas y el ingreso fue de 3 días (mínimo: 0 días, máximo: 17 días). De los 100 pacientes, 77 (77\%) tuvieron al menos un factor de riesgo para Influenza grave (tabla 2), incluyendo el $60,7 \%$ de los niños (< 18 años) y el $83,3 \%$ de los adultos ( $\geq 18$ años); el $46 \%$ tuvo al menos 2 factores y todos los pacientes con 65 o más años presentaron alguna condición médica de
Tabla 1 Características de 100 pacientes ingresados por infección con Influenza A H1N1 2009 en el hospital Ramón y Cajal (julio-diciembre 2009)

\begin{tabular}{lll}
\hline Característica & Número & Porcentaje \\
\hline $\begin{array}{l}\text { Mujer } \\
\text { Grupos de edad }\end{array}$ & 54 & 54,0 \\
0-23 meses & 8 & 8,0 \\
$2-4$ años & 8 & 8,0 \\
$5-9$ años & 9 & 9,0 \\
$10-17$ años & 3 & 3,0 \\
$18-49$ años & 39 & 39,0 \\
$50-64$ años & 22 & 22,0 \\
$\geq 65$ años & 11 & 11,0 \\
Procedencia & & \\
Madrid & 94 & 94,0 \\
Castilla y León & 2 & 2,0 \\
Castilla La Mancha & 1 & 1,0 \\
Barcelona & 1 & 1,0 \\
Turista extranjero & 2 & 2,0 \\
\hline a La mediana de edad de los pacientes fue 38 años (mínimo: 4 \\
meses, máximo: 80 años). \\
b Lugar de residencia habitual.
\end{tabular}

base. El asma fue la enfermedad más frecuente entre los niños $(28,6 \%)$; mientras en los adultos prevaleció el hábito tabáquico $(30,6 \%)$. Los trastornos convulsivos, la disfunción cognitiva y la enfermedad neuromuscular fueron más comunes entre los menores $(17,9 \%)$ que entre los adultos $(8,3 \%)$. Ninguna de las pacientes estaba embarazada en el momento del ingreso (tabla 2).

\section{Laboratorio clínico y radiografía de tórax}

Al ingreso, 15 de 96 pacientes con información del hemograma $(15,6 \%)$ tenían leucocitopenia, $24(25,0 \%)$ presentaban anemia, y $21(22,3 \%)$ trombocitopenia (tabla 3$)$. De los 48 pacientes con diagnóstico de neumonía, en 11 (22,0\%) se obtuvieron cultivos positivos. Cuatro en hemocultivos y 7 en esputo u otra muestra respiratoria. Streptococcus pneumoniae fue el microorganismo más frecuente $(36,3 \%)$, seguido por Pseudomonas aeruginosa $(27,2 \%)$, y cada uno de los siguientes: Staphylococcus coagulasa negativo, Haemophilus influenzae, Escherichia coli y Stenotrophomonas maltophilia, en 9,0\% de las muestras. En los 37 pacientes restantes no se aislaron microorganismos en las muestras analizadas.

De los 98 pacientes con radiografía de tórax al ingreso, $47(48,0 \%)$ presentaron algún infiltrado; la mediana de edad de estos pacientes fue 36 años (mínimo: 1 año, máximo: 80 años), y el $66,0 \%$ tuvo al menos un factor de riesgo. Los hallazgos radiológicos incluyeron infiltrados bilaterales en 22 pacientes $(46,8 \%)$ e infiltrados limitados a un lóbulo en $25(53,2 \%)$.

\section{Tratamiento}

De los 100 pacientes, $94(94,0 \%)$ recibieron tratamiento antiviral. De éstos, todos recibieron oseltamivir y $2(2,0 \%)$ 
Tabla 2 Factores de riesgo estratificados por edad

\begin{tabular}{|c|c|c|c|}
\hline Factor de riesgo & $\begin{array}{l}\text { Todos } \\
(\mathrm{N}=100)\end{array}$ & $\begin{array}{l}<18 \text { años } \\
(\mathrm{N}=28)\end{array}$ & $\begin{array}{l}\geq 18 \text { años } \\
(\mathrm{N}=72)\end{array}$ \\
\hline & \multicolumn{3}{|c|}{ número (\%) } \\
\hline Fumador actual & $22(22,0)$ & $0(0)$ & $22(30,6)$ \\
\hline Asma & $22(22,0)$ & $8(28,6)$ & $14(19,4)$ \\
\hline EPOC & $10(10,0)$ & $0(0)$ & $10(13,9)$ \\
\hline Otra enfermedad respiratoria crónica ${ }^{a}$ & $9(9,0)$ & $2(7,1)$ & $7(9,7)$ \\
\hline Diabetes & $11(11)$ & $0(0)$ & $11(15,3)$ \\
\hline Otras enfermedades metabólicas ${ }^{\mathrm{b}}$ & $12(12,0)$ & $3(10,7)$ & $9(12,5)$ \\
\hline Insuficicencia renal & $9(9,0)$ & $0(0)$ & $9(12,5)$ \\
\hline Inmunodeficiencia activa ${ }^{c}$ & $16(16,0)$ & $1(3,6)$ & $15(20,8)$ \\
\hline Cáncer & $9(9,0)$ & $2(7,1)$ & $7(9,7)$ \\
\hline Enfermedad cardiovascular ${ }^{d}$ & $15(15,0)$ & $2(7,1)$ & $13(18,1)$ \\
\hline Obesidad $^{e}$ & $12(12,0)$ & $0(0)$ & $12(16,7)$ \\
\hline Enfermedad hepática crónica & $4(4,0)$ & $1(3,6)$ & $3(4,2)$ \\
\hline Hemoglobinopatía y/o anemia & $4(4,0)$ & $1(3,6)$ & $3(4,2)$ \\
\hline Trastornos convulsivos & $7(7,0)$ & $3(10,7)$ & $4(5,6)$ \\
\hline Síndrome de Down/déficit cognitivo & $6(6,0)$ & $3(10,7)$ & $3(4,2)$ \\
\hline Enfermedad neuromuscular & $5(5,0)$ & $3(10,7)$ & $2(2,8)$ \\
\hline Tratamiento prolongado con ácido acetilsalicílico & $4(4,0)$ & $0(0)$ & $4(5,6)$ \\
\hline
\end{tabular}

oseltamvir seguido por zanamivir. En 80 pacientes para los cuales se contaba con información acerca de la fecha de inicio del tratamiento antiviral, la mediana de tiempo entre el comienzo de los síntomas y el inicio de la terapia fue 3 días (mínimo: 0 días, máximo: 18 días); 36 (45,0\%) recibie-

Tabla 3 Pruebas de laboratorio clínico

\begin{tabular}{|c|c|}
\hline Prueba & $\mathrm{N} / \mathrm{N}$ total $(\%)$ \\
\hline Leucopenia $\left(<5.000\right.$ leucocitos $\left.x \mathrm{~mm}^{3}\right)$ & $15 / 96(15,6)$ \\
\hline Leucocitosis $\left(>11000 \text { leucocitos } x \mathrm{~mm}^{3}\right)^{\mathrm{a}}$ & $24 / 96(25,0)$ \\
\hline Anemia $^{\mathrm{b}}$ & $24 / 96(25,0)$ \\
\hline $\begin{array}{l}\text { Trombocitopenia }(<150.000 \text { plaquetas } x \\
\mathrm{mm}^{3} \text { ) }\end{array}$ & $21 / 96(21,9)$ \\
\hline Trombocitosis (> 350.000 plaquetas $\times \mathrm{mm}^{3}$ ) & $7 / 96(7,3)$ \\
\hline \multicolumn{2}{|l|}{ Alanino aminotransferasa elevada ${ }^{c}$} \\
\hline Cualquier elevación & $29 / 79(36,7)$ \\
\hline$\geq 2 \times$ el límite superior del rango normal & ,7) \\
\hline \multicolumn{2}{|l|}{ Aspartato aminotransferasa ${ }^{\mathrm{d}}$} \\
\hline Cualquier elevación & $30 / 79(37,9)$ \\
\hline$\geq 2 \times$ el límite superior del rango normal & $14 / 79(17,7)$ \\
\hline Bilirrubina total elevada (>1,2 mg/dl) & $4 / 79(5,1)$ \\
\hline \multicolumn{2}{|c|}{ 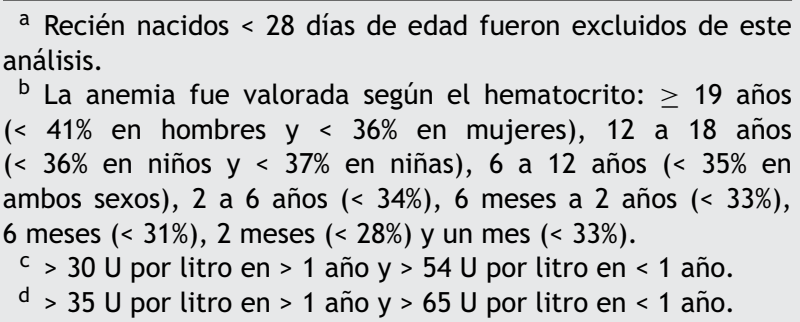 } \\
\hline
\end{tabular}

ron el antiviral en las primeras $48 \mathrm{~h}$ después del inicio de los síntomas, en $5(6,3 \%)$ se administró la terapia antes del ingreso en el hospital, en $70(87,5 \%)$ se hizo durante las primeras $48 \mathrm{~h}$ del ingreso y en $5(6,3 \%)$ después de $48 \mathrm{~h}$ de haber sido ingresados. La mediana de duración del tratamiento con oseltamivir fue 5 días (mínimo: 5 días, máximo: 22 días) y 36 días para el zanamivir (mínimo: 6 días, máximo: 67 días).

\section{Análisis de la severidad de la enfermedad}

De los 100 pacientes analizados, 19 fueron ingresados en la $\mathrm{UCl}$ y 2 fallecieron por gripe. El 79\% de los admitidos a dicha unidad presentaron al menos un factor de riesgo. Diez de éstos $(52,6 \%)$ requirieron ventilación mecánica, 7 (36,8\%) desarrollaron un síndrome de dificultad respiratoria aguda (SDRA) y $3(15,8 \%)$ tuvieron un diagnóstico clínico de sepsis. Todos los pacientes recibieron tratamiento antiviral con un patrón de administración que no varió respecto al descrito anteriormente.

El análisis de la estancia hospitalaria mostró que la mediana de los pacientes admitidos en $\mathrm{UCI}$ fue 7 días mayor que la observada en los pacientes ingresados en planta; esta diferencia fue estadísticamente significativa $(p<0,001)$.

El primer fallecimiento por gripe pandémica se produjo en una niña de 14 años de edad con síndrome de LennoxGastaut que desarrolló un síndrome séptico con coagulopatía de consumo secundario a infección respiratoria aguda por Influenza A/H1N1 2009 y posible sobreinfección bacteriana. El segundo fallecimiento ocurrió en un hombre de 60 años sin factores de riesgo conocidos para complicación por gripe, ingresado en la $\mathrm{UCl}$ por insuficiencia respiratoria aguda secundaria a neumonía por Influenza A/H1N1 2009, el cual 
Tabla 4 Análisis bivariado de las características clínicas y los factores de riesgo entre pacientes que no fueron ingresados en $\mathrm{UCI}$ y quienes sí fueron ingresados en esta unidad

\begin{tabular}{|c|c|c|c|}
\hline Característica & $\begin{array}{l}\text { Paciente que no } \\
\text { ingresaron en } \mathrm{UCl} \\
(\mathrm{N}=81)\end{array}$ & $\begin{array}{l}\text { Pacientes que } \\
\text { ingresaron en } \mathrm{UCl} \\
(\mathrm{N}=19)\end{array}$ & $\mathrm{p}$ \\
\hline \multicolumn{4}{|l|}{ Edad } \\
\hline Mediana, años (mínimo-máximo) & $38(0-80)$ & $46(1-72)$ & $0,253^{a}$ \\
\hline$<18$ años, n. ${ }^{\circ}(\%)$ & $23(28,4)$ & $5(26,3)$ & $0,856^{\mathrm{b}}$ \\
\hline Oxígeno suplementario, $n .^{\circ} / n .^{\circ}$ total (\%) & $44 / 78(56,4)$ & $14 / 19(73,7)$ & $0,168^{b}$ \\
\hline $\begin{array}{l}\text { Oximetría de pulso < } 95 \% \text { con aire ambiental, } n .{ }^{\circ} / n .^{\circ} \\
\text { total (\%) }\end{array}$ & $43 / 79(54,4)$ & $13 / 19(68,4)$ & $0,269^{b}$ \\
\hline Enfermedad respiratoria crónica, $n .^{\circ}(\%)^{c}$ & $34(42,0)$ & $4(21,1)$ & $0,091^{\mathrm{b}}$ \\
\hline Enfermedad metabólica, $n \cdot{ }^{\circ}(\%)^{d}$ & $13(16,0)$ & $8(42,1)$ & $0,012^{\mathrm{e}}$ \\
\hline Insuficiencia renal & $7(8,6)$ & $2(10,5)$ & $0,796^{\mathrm{b}}$ \\
\hline Inmunodeficiencia activa / cáncer, $n .^{\circ}(\%)$ & $16(19,8)$ & $3(15,8)$ & $1,000^{e}$ \\
\hline Enfermedad cardiovascular, n. ${ }^{\circ}(\%)$ & $9(11,1)$ & $6(31,6)$ & $0,036^{e}$ \\
\hline Trastorno neurocognitivo/neuromuscular, $n .^{\circ}(\%)^{f}$ & $9(11,1)$ & $2(10,5)$ & $1,000^{e}$ \\
\hline Obesidad, $n .^{\circ}(\%)^{\mathrm{g}}$ & $10(12,3)$ & $2(10,5)$ & $1,000^{e}$ \\
\hline Infiltrados en la radiografía de tórax, $n .{ }^{\circ} / n .^{\circ}$ total (\%) & $34 / 79(43,0)$ & $13 / 19(68,4)$ & $0,047^{b}$ \\
\hline \multicolumn{4}{|l|}{ Tratamiento antiviral } \\
\hline Sí, n. ${ }^{\circ}(\%)$ & $75(92,6)$ & $19(100,0)$ & $0,592^{\mathrm{e}^{* *}}$ \\
\hline $\begin{array}{l}\leq 2 \text { días después del inicio de los síntomas, } n .^{\circ} / \mathrm{n} .^{\circ} \\
\text { total (\%) }\end{array}$ & $29 / 63(46,0)$ & $7 / 17(41,2)$ & $0,721^{\mathrm{b}}$ \\
\hline $\begin{array}{l}\text { Días entre el comienzo de los síntomas y el inicio de la } \\
\text { terapia, mediana (mínimo-máximo) }\end{array}$ & $3(0-18)$ & $4(0-10)$ & $0,082^{\mathrm{a}}$ \\
\hline \multicolumn{4}{|l|}{$\begin{array}{l}\text { a U de Mann-Whitney. } \\
\text { b Ji cuadrado. } \\
\text { c Incluye asma, EPOC u otra enfermedad respiratoria crónica. } \\
\text { d Incluye diabetes. } \\
\text { e Prueba exacta de Fisher. } \\
\text { f Incluye trastornos convulsivos, síndrome de Down u otra disfu }\end{array}$} \\
\hline
\end{tabular}

desarrolló hipoxia grave con oliguria e hipotensión. Ambos pacientes recibieron tratamiento antiviral en el momento del ingreso en el hospital, la niña durante las primeras $48 \mathrm{~h}$ de iniciados los síntomas y el adulto 7 días después.

En el análisis bivariado, los pacientes ingresados en la UCI presentaron mayor probabilidad de tener una enfermedad metabólica, incluida diabetes, y algún infiltrado en la radiografía de tórax al ingreso que los pacientes no admitidos a esta unidad (tabla 4). En cambio, no se encontró diferencia en la mediana de tiempo entre el inicio de los síntomas y el ingreso en el hospital entre estos pacientes. Ambos grupos tampoco mostraron diferencia respecto a haber recibido vacunación para Influenza estacional 2008-2009. En un análisis multivariante que consideró como modelo máximo enfermedad metabólica, presencia de infiltrados en la radiografía de tórax de ingreso y enfermedad cardiovascular, las 2 primeras variables se asociaron de forma significativa a ingreso en $\mathrm{UCl}$.

\section{Discusión}

A diferencia de las epidemias de Influenza estacional que causan tasas de hospitalización más altas en población mayor de 65 años de edad y menor de 2 años, la incidencia de hospitalización por gripe pandémica en este estudio fue mayor en los menores de 10 años de edad ${ }^{14}$. La tasa de hospitalización en mayores de 65 años fue comparable a la del grupo con menor incidencia, de 10 a 17 años de edad. Las tasas estimadas podrían infraestimar la verdadera incidencia de hospitalización por gripe pandémica, si se tiene en cuenta que no incluyen en el numerador los casos de gripe con domicilio en el Área IV e ingresados en un hospital de otra área sanitaria diferente de la comunidad de Madrid. La ausencia de embarazadas en este estudio se debe a que no existe un servicio de obstetricia en el hospital y las embarazadas que precisaban ingreso fueron derivadas a otro hospital de referencia.

Al igual que en otras series publicadas ${ }^{17,18,24}$, la mayor proporción de ingresos por gripe pandémica en nuestro centro afectó a población joven entre 18 y 49 años, constituyendo los menores de 65 años cerca del $90 \%$ de todos los ingresos. Esta discrepancia entre la tasa de incidencia de hospitalización y la proporción de ingresos por edad está explicada por la distribución poblacional del Área IV, donde un $47 \%$ de ésta tiene entre 18 y 49 años de edad y tan sólo el $10 \%$ es menor de 10 años.

Más del $80 \%$ de los adultos y del $60 \%$ de los niños presentaron algún factor de riesgo asociado a Influenza estacional grave. La prevalencia de estos factores en nuestra serie es superior a la encontrada por otros autores, tanto en adultos como en niños ingresados por Influenza estacional ${ }^{15,25-27}$. 
Esta diferencia puede ser explicada por una tendencia razonable entre los médicos a ingresar preferentemente a los pacientes con factores de riesgo para complicación por gripe. Al igual que en la gripe estacional, la enfermedad respiratoria crónica, la EPOC y el asma fueron en conjunto los factores de riesgo más frecuentes en los pacientes ingresados.

Una revisión de la literatura médica señala que la administración temprana de oseltamivir en pacientes con infección por virus pandémico (H1N1) 2009 puede reducir la duración de la hospitalización y el riesgo de progresión a enfermedad grave que requiera ingreso en la $\mathrm{UCI}$ o desencadene la muerte ${ }^{13}$. Hay evidencia de que la terapia antiviral administrada en las primeras $48 \mathrm{~h}$ del comienzo de los síntomas produce un mayor beneficio ${ }^{13}$. En nuestro análisis no se encontró que los pacientes hospitalizados en planta tuvieran una mayor probabilidad de haber recibido oseltamivir en las primeras $48 \mathrm{~h}$ del comienzo de la enfermedad que los admitidos en $\mathrm{UCl}$ por gripe.

Un índice de masa corporal $(I M C) \geq 28$ ha sido implicado como factor de riesgo para gripe pandémica grave ${ }^{12,15,17-19,22,23}$. Adicionalmente, está asociado a otras comorbilidades que son factores de riesgo conocidos para Influenza complicada, tales como diabetes, enfermedades metabólicas, enfermedad cardiovascular y pulmonar (apnea obstructiva del sueño y síndrome de hipoventilación y obesidad). En este estudio, la ausencia de asociación estadística entre obesidad y enfermedad grave puede ser explicada por el número restringido de eventos analizados. Sin embargo, los resultados del análisis multivariante son concordantes con aquellos estudios que vinculan las enfermedades metabólicas como factores de riesgo asociados a Influenza grave $^{12,28,29}$. La presencia de infiltrados en la radiografía de tórax también ha sido asociada de forma independiente a gripe pandémica grave en la literatura médica ${ }^{17}$. En esta serie, el $\mathbf{9 8 \%}$ de los pacientes con este hallazgo radiológico fueron diagnosticados con neumonía, y el $75 \%$ de los ingresados en la UCI presentaron esta complicación.

Como sucede en otros estudios, en nuestros pacientes una radiografía de tórax anormal en el momento del ingreso, junto con la presencia de ciertas comorbilidades, especialmente enfermedades metabólicas, sugieren la posibilidad de una evolución más grave de la enfermedad.

\section{Conflicto de intereses}

Los autores declaran no tener ningún conflicto de intereses.

\section{Bibliografía}

1. Centers for Disease Control and Prevention. Outbreak of swineorigin influenza A (H1N1) virus infection-Mexico, March-April 2009. MMWR. 2009; 58: 467-70.

2. Fajardo-Dolci GE, Hernandez-Torres F, Santacruz-Varela J, Rodriguez-Suarez J, Lamy P, Arboleya-Casanova H, et al. Epidemiological profile of mortality due to human influenza A (H1N1) in Mexico. Salud Publica Mex. 2009;51:361-71.

3. Centers for Disease Control and Prevention. Swine influenza A (H1N1) infection in two children-Southern California, MarchApril 2009. MMWR. 2009; 58: 400-2.

4. Santa-Olalla PP, Cortes GM, Martinez Sanchez EV, Nogareda MF, Limia SA, Pachon DAl, et al. Enhanced surveillance of initial cases of pandemic influenza (H1N1) 2009 infection in Spain, April-June 2009. Rev Esp Salud Publica. 2010;84:529-46.

5. Sierra Moros MJ, Vazquez TM, Santa-Olalla PP, Limia SA, Cortes GM, Pachon DAl. Epidemiological surveillance activities during the 2009 influenza pandemic in Spain: lessons learnt one year after. Rev Esp Salud Publica. 2010;84:463-79.

6. Centers for Disease Control and Prevention. Hospitalized patients with novel influenza A (H1N1) virus infection - California, April-May, 2009. MMWR 2009;58:536-41.

7. Baker MG, Wilson N, Huang QS, Paine S, Lopez L, Bandaranayake D, et al. Pandemic influenza A(H1N1)v in New Zealand: the experience from April to August 2009. Euro Surveill. 2009;14, pii= 19319. Disponible en:http://www.eurosurveillance.org/ ViewArticle.aspx?Articleld=19319.

8. Dawood FS, Jain S, Finelli L, Shaw MW, Lindstrom S, Garten RJ, et al. Emergence of a novel swine-origin influenza A (H1N1) virus in humans. N Engl J Med. 2009;360:2605-15.

9. Donaldson LJ, Rutter PD, Ellis BM, Greaves FE, Mytton OT, Pebody RG, et al. Mortality from pandemic A/H1N1 2009 influenza in England: public health surveillance study. BMJ. 2009;339:b5213.

10. Garske T, Legrand J, Donnelly CA, Ward H, Cauchemez S, Fraser $C$, et al. Assessing the severity of the novel influenza A/H1N1 pandemic. BMJ. 2009;339:b2840.

11. Presanis AM, de Angelis D, Hagy A, Reed C, Riley S, Cooper BS, et al. The severity of pandemic H1N1 influenza in the United States, from April to July 2009: a Bayesian analysis. PLoS Med. 2009;6:e1000207.

12. Vaillant L, La Ruche G, Tarantola A, Barboza P, for the epidemic intelligence team at InVS. Epidemiology of fatal cases associated with pandemic H1N1 influenza 2009. Euro Surveill. 2009;14:33, pii=19309. Disponible en: http:// www.eurosurveillance.org/ViewArticle. aspx?Articleld=19309.

13. Bautista E, Chotpitayasunondh T, Gao Z, Harper SA, Shaw M, Uyeki TM, et al. Clinical aspects of pandemic 2009 influenza A (H1N1) virus infection. N Engl J Med. 2010;362:1708-19.

14. To KK, Wong SS, Li IW, Hung IF, Tse H, Woo PC, et al. Concurrent comparison of epidemiology, clinical presentation and outcome between adult patients suffering from the pandemic influenza $A$ (H1N1) 2009 virus and the seasonal influenza $A$ virus infection. Postgrad Med J. 2010;86:515-21.

15. Louie JK, Acosta M, Winter K, Jean C, Gavali S, Schechter R, et al. Factors associated with death or hospitalization due to pandemic 2009 influenza A(H1N1) infection in California. JAMA. 2009;302:1896-902.

16. Miller III RR, Markewitz BA, Rolfs RT, Brown SM, Dascomb KK, Grissom CK, et al. Clinical findings and demographic factors associated with ICU admission in Utah due to novel 2009 influenza A(H1N1) infection. Chest. 2010;137:752-8.

17. Nguyen-Van-Tam JS, Openshaw PJ, Hashim A, Gadd EM, Lim WS, Semple MG, et al. Risk factors for hospitalisation and poor outcome with pandemic A/H1N1 influenza: United Kingdom first wave (May-September 2009). Thorax. 2010;65:645-51.

18. Cui W, Zhao H, Lu X, Wen Y, Zhou Y, Deng B, et al. Factors associated with death in hospitalized pneumonia patients with 2009 H1N1 influenza in Shenyang, China. BMC Infect Dis. 2010;10:145.

19. Santa-Olalla PP, Cortes GM, Limia SA, Andres PJ, Pachon DAI, Sierra Moros MJ. Critically ill patients with 2009 pandemic influenza A (H1N1) infection in Spain: factors associated with death April 2009-January 2010. Rev Esp Salud Publica. 2010;84:547-67.

20. Louie JK, Acosta M, Samuel MC, Schechter R, Vugia DJ, Harriman $\mathrm{K}$, et al. A novel risk factor for a novel virus: obesity and 2009 pandemic influenza A (H1N1). Clin Infect Dis. 2011;52:301-12.

21. Yu H, Feng Z, Uyeki TM, Liao Q, Zhou L, Feng L, et al. Risk factors for severe illness with 2009 pandemic Influenza 
A (H1N1) virus infection in China. Clin Infect Dis. 2011;52: 457-65.

22. Rello J, Rodriguez A, Ibanez P, Socias L, Cebrian J, Marques A, et al. Intensive care adult patients with severe respiratory failure caused by Influenza A (H1N1)v in Spain. Crit Care. 2009;13:R148.

23. Rodriguez A, Socias L, Guerrero JE, Figueira JC, Gonzalez N, Maravi-Poma E, et al., GETGAG/SEMICYUC/(Spanish Working Group on Severe Pandemic Influenza A/SEMICYUC). Pandemic influenza $A$ in the ICU: experience in Spain and Latin America. Med Intensiva. 2010;34:87-94.

24. Jain S, Kamimoto L, Bramley AM, Schmitz AM, Benoit SR, Louie J, et al. Hospitalized patients with 2009 H1N1 influenza in the United States, April-June 2009. N Engl J Med. 2009;361:1935-44.

25. Belongia EA, Irving SA, Waring SC, Coleman LA, Meece JK, Vandermause $M$, et al. Clinical characteristics and 30-day outcomes for influenza A 2009 (H1N1), 2008-2009 (H1N1), and 2007-2008 (H3N2) infections. JAMA. 2010;304: 1091-8.

26. McGeer A, Green KA, Plevneshi A, Shigayeva A, Siddiqi N, Raboud J, et al. Antiviral therapy and outcomes of influenza requiring hospitalization in Ontario, Canada. Clin Infect Dis. 2007;45:1568-75.

27. Schrag SJ, Shay DK, Gershman K, Thomas A, Craig AS, Schaffner W, et al. Multistate surveillance for laboratoryconfirmed, influenza-associated hospitalizations in children: 2003-2004. Pediatr Infect Dis J. 2006;25:395-400.

28. Allard R, Leclerc $P$, Tremblay C, Tannenbaum TN. Diabetes and the severity of pandemic influenza A (H1N1) infection. Diabetes Care. 2010;33:1491-3.

29. Haglin LM, Burman LA, Nilsson M. Predisposing chronic diseases and hypophosphatemia in patients with influenza. Arch Gerontol Geriatr. 2010;51:26-30. 